\title{
Spectral geometry for the standard model without fermion doubling
}

\author{
Arkadiusz Bochniak $\odot$ and Andrzej Sitarz $\odot$ \\ Institute of Theoretical Physics, Jagiellonian University, prof. Stanistawa Łojasiewicza 11, \\ 30-348 Kraków, Poland
}

(Received 21 January 2020; accepted 30 March 2020; published 20 April 2020)

\begin{abstract}
We propose a simple model of noncommutative geometry to describe the structure of the standard model, which satisfies $\operatorname{spin}_{c}$ condition, has no fermion doubling, does not lead to the possibility of color symmetry breaking, and explains the $C P$ violation as the failure of the reality condition for the Dirac operator.
\end{abstract}

DOI: 10.1103/PhysRevD.101.075038

\section{INTRODUCTION}

The standard model of particle interactions is certainly one of the most successful and one of the best tested theories about the fundamental constituents of matter and the forces between them. Even though we still have no satisfactory description of the strong interactions in the low-energy regime and there are some puzzles concerning masses and the character of neutrinos as well as there are some experimental signs that could point out to new physics, the standard model appears to be robust and verified. Yet neither the content of the fermion sector, the mixing between the families, nor the fundamentally different character of the Higgs boson from other gauge bosons appears to have a satisfactory geometrical explanation.

One of the few theories that aimed to provide a sound geometrical basis for the structure of the standard model, explaining the appearance of the Higgs and symmetrybreaking potential, was noncommutative geometry (see Refs. [1-3]). It was constructed with the core idea that spaces with points can be replaced with algebras and provided a plausible explanation of the gauge group of the standard model and the particles in its representation as linked to the unitary group of a finite-dimensional algebra. Merged with the Kaluza-Klein idea that the physical spacetime has extra dimensions, the geometry of the finite-dimensional algebra (in the noncommutative sense) gave rise to the Higgs field understood as a connection, and the Higgs symmetry-breaking potential appeared as the usual Yang-Mills term in the action.

Published by the American Physical Society under the terms of the Creative Commons Attribution 4.0 International license. Further distribution of this work must maintain attribution to the author(s) and the published article's title, journal citation, and DOI. Funded by SCOAP ${ }^{3}$.
The original model, which is based on the construction of a product geometry, with the resulting geometry being the tensor product of a usual "commutative" space with the finite-dimensional noncommutative geometry suffers from two problems. First, in the original formulation, it is Euclidean. Second, the product structure leads to the quadrupling of the degrees of freedom in the classical Lagrangian [4,5]. Moreover, the conditions put on the Dirac operator for the finite geometry are not sufficient to restrict the class of possible operators to the physical one, leaving the possibility for the nonphysical $S U(3)$-breaking geometries [6-9]. Though the latter problems appear to have at least a partial solution [8], we believe that they can be completely avoided if the noncommutative geometry behind the standard model is assumed to be $\operatorname{spin}_{c}$ only.

It is worth noting that, in addition to the aforementioned issues in formulating the noncommutative standard model, there is also one significant problem related to the disagreement in the predicted Higgs mass and its experimental value [3]. Furthermore, there is also an accompanying problem related to the low value of the Higgs, known as the Higgs vacuum instability. Several possible solutions have been proposed to fix these problems, starting from adding new fermions $[10,11]$, introducing an additional scalar field (so-called $\sigma$ field) [12,13], extending the algebra to the Grand Symmetry models, $[14,15]$ or using twisted spectral triples formulation [16,17]. All of the mentioned extensions are still based on the concept of real spectral triples with the product geometry, and they similarly require cutting down the quadrupled number of the degrees of freedom.

In what follows, we present a $\operatorname{spin}_{c}$ description of the geometry for the standard model, which does not require fermion doubling and satisfies the $\operatorname{spin}_{c}$ duality for spinors provided that the mass matrices and mixing matrices are nondegenerate. The crucial role is then played not by the Lorentzian Dirac operator but rather by its Krein-shift $\tilde{D}$, the product of the Krein space fundamental symmetry $\beta$ and the Dirac operator $D$. This operator can be understood 
as the self-adjoint component of the Krein decomposition of the Lorentzian Dirac operator, $D=\beta \tilde{D}$. Moreover, we link the breaking of the $J$ condition between the real structure and the Dirac operator to the appearance of the $C P$-symmetry breaking in the standard model.

We have to stress that the approach we take is based on the physical Lagrangian of the standard model and it is aiming to put a geometrical meaning to its form and the major features like the lack of strong symmetry breaking or $C P$ violation. Our interpretation, based on the noncommutative $\operatorname{spin}_{c}$ geometry, explains both phenomena, and we consider it as a strong signal to take this model seriously. At present, we cannot provide a precise quantitative result for the Higgs mass in our approach, which may be compared with the experimental value. The explicit spectral action computations for the model are currently in progress [18], and for a next step, we aim to see whether the extended models (with Grand Symmetry additional fermions or a scalar field) will satisfy the $\operatorname{spin}_{c}$ condition.

\section{DIRAC OPERATOR FOR THE STANDARD MODEL}

The Dirac operator for the four-dimensional Minkowski space is of the form $D=i \gamma^{\mu} \partial_{\mu}$, with the gamma matrices satisfying the relation $\gamma^{\mu} \gamma^{\nu}+\gamma^{\nu} \gamma^{\mu}=2 \eta^{\mu \nu}$, where $\eta^{\mu \nu}$ is the standard Minkowski metric of signature $(+,-,-,-)$. We use the conventions of [8], so that $\gamma^{0}$ is self-adjoint and the remaining gamma matrices are anti-self-adjoint.

The Lorentz-invariant fermionic action, which leads to the Dirac equation, is

$$
\int_{M} \bar{\psi} D \psi=\int_{M} \psi^{\dagger} \tilde{D} \psi
$$

where $\bar{\psi}=\psi^{\dagger} \gamma^{0}$ and $\tilde{D}=\gamma^{0} D$. The operator, $\tilde{D}$, is a symmetric operator, which we call the Krein shift of the Dirac operator. This follows from the properties of the Lorentzian Dirac operator $D$, which is Krein self-adjoint [19], $D^{\dagger}=\gamma^{0} D \gamma^{0}$, where $\gamma^{0}$ is the fundamental symmetry of the Krein space. Written explicitly in the chiral representation, it becomes

$$
\tilde{D}=i\left(\begin{array}{cc}
\sigma^{\mu} & 0 \\
0 & \tilde{\sigma}_{\mu}
\end{array}\right) \partial_{\mu},
$$

where $\sigma^{\mu}$ and $\tilde{\sigma}^{\mu}$ are the standard and associated Pauli matrices, $\tilde{\sigma}^{0}=\sigma^{0}, \tilde{\sigma}^{k}=-\sigma^{k}$.

The Lorentzian Dirac operator and the related Lorentzian spectral triple have the standard $\mathbb{Z}_{2}$ grading $\gamma$ and the charge conjugation operator given,

$\gamma=\left(\begin{array}{cc}1_{2} & 0 \\ 0 & -1_{2}\end{array}\right), \quad \mathcal{J}=i \gamma^{2} \circ c c=i\left(\begin{array}{cc}0 & \sigma^{2} \\ -\sigma^{2} & 0\end{array}\right) \circ c c$,

where $c c$ denotes the usual complex conjugation of spinors. The operators $D, \gamma, \mathcal{J}$ satisfy the usual commutation relations for the geometry of the signature $(1,3)$,
$D \gamma=-\gamma D, \quad D \mathcal{J}=\mathcal{J} D, \quad \mathcal{J}^{2}=1, \quad \mathcal{J} \gamma=-\gamma \mathcal{J}$,

whereas for the Krein-shifted operator, we have

$\tilde{D} \gamma=\gamma \tilde{D}, \quad \tilde{D} \mathcal{J}=-\mathcal{J} \tilde{D}, \quad \mathcal{J}^{2}=1, \quad \mathcal{J} \gamma=-\gamma \mathcal{J}$.

The so-far accepted and tested experimentally action for the standard model of fundamental interactions can be viewed as the extension of the action for a single bispinor to a family of particles, with the additional terms in the action arising from a slight modification of the Dirac operator by an endomorphism of the finite-dimensional space of fermions.

Before we discuss this extension and the conditions it satisfies, we recall the notion of Riemannian spectral triples and $\operatorname{spin}_{c}$-spectral triples, which form a bigger class than these arising from generalization of the spin geometry only.

\section{RIEMANNIAN AND PSEUDO-RIEMANNIAN SPECTRAL TRIPLES}

A Riemannian finite spectral triple [20] built over a finite-dimensional algebra $A$ is a collection of data $\left(A, D, H, \pi_{L}, \pi_{R}\right)$, where $\pi_{L}$ is the representation of $A$ on $H$ and $\pi_{R}$ is the representation of $A^{o p}$ (the opposite algebra to $A$ ) on $H$ such that

$$
\begin{gathered}
{\left[\pi_{L}(a), \pi_{R}(b)\right]=0,} \\
{\left[\left[D, \pi_{L}(a)\right], \pi_{R}(b)\right]=0,}
\end{gathered}
$$

for all $a \in A$ and $b \in A^{o p}$.

We say that the spectral triple is of $\operatorname{spin}_{c}$ (see Ref. [21] and compare with the classical result [22]) type if

$$
\left(C l_{D}\left(\pi_{L}(A)\right)^{\prime}=\pi_{R}(A)\right.
$$

or of Hodge type if

$$
\left(C l_{D}\left(\pi_{L}(A)\right)^{\prime}=C l_{D}\left(\pi_{R}(A)\right) .\right.
$$

By the generalized Clifford algebra $C l_{D}\left(\pi_{L}(A)\right)$ [and similarly $\left.C l_{D}\left(\pi_{R}(A)\right)\right]$, we understand the algebra generated by $\pi_{L}(a)$ and $\left[D, \pi_{L}(b)\right]$ for all $a, b \in A$.

Of course, genuine Riemannian geometries require further assumption that the operator $D$ has a compact resolvent. In the case of Lorentzian or, more generally, pseudo-Riemannian geometries, we might follow the path of Ref. [19], extending the definition of Lorentzian real spectral triples to Lorentzian $\operatorname{spin}_{c}$ geometries.

\section{FERMIONS AND THE ALGEBRA OF THE STANDARD MODEL}

Let us recall a convenient parametrization of the particle content in the one-generation standard model [21], 


$$
\Psi=\left(\begin{array}{cccc}
\nu_{R} & u_{R}^{1} & u_{R}^{2} & u_{R}^{3} \\
e_{R} & d_{R}^{1} & d_{R}^{2} & d_{R}^{3} \\
\nu_{L} & u_{L}^{1} & u_{L}^{2} & u_{L}^{3} \\
e_{L} & d_{L}^{1} & d_{L}^{2} & d_{L}^{3}
\end{array}\right) \in M_{4}\left(H_{W}\right)
$$

where each of the entries is the Weyl spinor over the Minkowski space with a fixed chirality. For the algebra $\mathcal{A}$, we take the algebra of functions over the Minkowski space, valued in $\mathbb{C} \oplus \mathbb{U} \oplus M_{3}(\mathbb{C})$, and chose the two representations of the algebra

$$
\pi_{L}(\lambda, q, m) \Psi=\left(\begin{array}{ccc}
\lambda & & \\
& \bar{\lambda} & \\
& & q
\end{array}\right) \Psi, \quad \pi_{R}(\lambda, q, m) \Psi=\Psi\left(\begin{array}{ll}
\lambda & \\
& m^{T}
\end{array}\right),
$$

where $\lambda, q$, and $m$ are complex, quaternion, and $M_{3}(\mathbb{C})$ valued functions, respectively. The representation $\pi_{L}$ acts by multiplying $\Psi$ from the left, whereas $\pi_{R}$ acts by multiplying $\Psi$ from the right. This is the reason that we transpose $m$ so that $\pi_{R}$ is indeed a representation. Observe that, since left and right multiplication commute, then $\left[\pi_{L}(a), \pi_{R}(b)\right]=0$ for all $a, b \in \mathcal{A}$; i.e., the zero-order condition is satisfied. Because of the simplicity of the notation at every point of the Minkowski space, we can encode any linear operator on the space of particles as an operator in $M_{4}(\mathbb{C}) \otimes M_{2}(\mathbb{C}) \otimes M_{4}(\mathbb{C})$, where the first and the last matrices act by multiplication from the left and from the right and the middle $M_{2}(\mathbb{C})$ matrix acts on the components of the Weyl spinor.

The full Lorentzian Dirac operator of the standard model is, in this notation, of the form

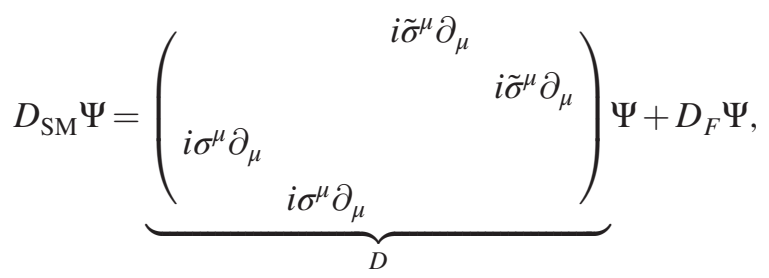

where $D_{F}$ is a finite endomorphism of the Hilbert space $M_{4}\left(H_{W}\right)$.

First of all, observe that the spatial part $D$ is covariant under the Lorentz transformations so that the Lagrange density (1) is invariant. Indeed, using the $S L(2, \mathbb{C})$ representation of the Lorentz group with an appropriate transformation of the Weyl spinors, it is obvious that $D$ transforms covariantly. On the other hand, $D_{F}$ will transform covariantly, so the full fermionic action will remain invariant under Lorentz transformations, only if it is an element of $M_{4}(\mathbb{C}) \otimes$ id $\otimes M_{4}(\mathbb{C})$, so it is a scalar from the point of view of Lorentz transformations.

At this point, it is the Lorentz invariance and the requirement that $D_{F}$ behaves like a scalar under Lorentz transformations that fixes $D_{F}$ to commute with the chirality $\Gamma$, which, in fact, can be written as an element of the algebra of the standard model, $\Gamma=\pi_{L}(1,-1,1)$. In the end, we have the genuine Lorentzian Dirac operator $D$ that anticommutes with $\Gamma$ and the finite part of the full Dirac operator, $D_{F}$, commuting with $\Gamma$, whereas the Krein-shifted parts have the opposite behavior.

Next, we find sufficient conditions for the Krein-shifted operator $\widetilde{D_{\mathrm{SM}}}$ to satisfy the first-order condition for the given algebra and the chosen representation. First, observe that $\tilde{D}$ alone obviously satisfies the order-one condition and therefore we need to check only $\widetilde{D_{F}}$. Suppose then that

$$
\left[\left[\widetilde{D_{F}}, \pi_{L}(a)\right], \pi_{R}(b)\right]=0,
$$

for all $a, b \in \mathcal{A}$. As any element in $\pi_{L}(\mathcal{A})$ commutes with $\pi_{R}(\mathcal{A})$, it suffices to find all $\tilde{D_{F}}$ that are self-adjoint, commute with the elements from $\pi_{R}(\mathcal{A})$, and anticommute with $\Gamma$. It is easy to see that such operators are restricted to

$\widetilde{D_{F}}=\underbrace{\left(\begin{array}{c}M_{l} \\ M_{l}^{\dagger}\end{array}\right)}_{D_{l}} \otimes e_{11}+\underbrace{\left(\begin{array}{c}M_{q} \\ M_{q}^{\dagger}\end{array}\right)}_{D_{q}} \otimes\left(1_{4}-e_{11}\right)$,

where $M_{l}, M_{q} \in M_{2}(\mathbb{C})$.

\section{A. $\operatorname{spin}_{c}$ condition}

The Krein-shifted Dirac operator satisfies first-order condition, yet it still may not provide the $\operatorname{spin}_{c}$ spectral geometry. We shall look for necessary and sufficient conditions that the commutant of the (complexified) Clifford algebra, $C l_{D}\left(\pi_{L}(\mathcal{A})\right)$, generated by $\pi_{L}(\mathcal{A})$ and $\left[\widetilde{D_{\mathrm{SM}}}, \pi_{L}(\mathcal{A})\right]$ is $\pi_{R}(\mathcal{A})$. First, observe that all operators in the so-defined $C l_{D}\left(\pi_{L}(\mathcal{A})\right)$ are endomorphisms of the space $M_{4}\left(H_{W}\right)$, which contain a subalgebra generated by the commutators of $\tilde{D}$ with functions $C_{\mathbb{C}}^{\infty}(M)$. This subalgebra acts on the Weyl spinors pointwise and can be identified with $M_{2}(\mathbb{C}) \oplus M_{2}(\mathbb{C})$-valued functions on the Minkowski space. The resulting subalgebra of the Clifford algebra acts only on the Weyl-spinorial components, separately in the left and in the right sectors. The commutant of this algebra in the endomorphisms of the Hilbert $M_{4}\left(H_{W}\right)$ space is then contained in the $M_{4}(\mathbb{C}) \otimes \mathrm{id} \otimes$ $M_{4}(\mathbb{C})$ (at each point of the Minkowski space).

Further, consider the subalgebra generated by the commutators of $\widetilde{D_{F}}$ with constant functions in $\mathcal{A}$. It is a subalgebra of $M_{4}(\mathbb{C}) \otimes \mathrm{id} \otimes\left(\mathbb{C} \oplus \mathbb{C}^{(3)}\right)$-valued constant functions over the Minkowski space, and it is easy to see that both subalgebras generate the full Clifford algebra. Therefore, the common commutant of both parts will be the commutant of the full Clifford algebra.

From the decomposition, it is easy to see that the commutant of the second part is the functions in 
id $\otimes M_{2}(\mathbb{C}) \otimes\left(\mathbb{C} \oplus M_{3}(\mathbb{C})\right)$ and therefore the common parts are functions valued in id $\otimes$ id $\otimes\left(\mathbb{C} \oplus M_{3}(\mathbb{C})\right)$, which indeed is the algebra $\pi_{R}(\mathcal{A})$.

\section{B. Three generations}

Let us consider three families of leptons and quarks, that is, the Hilbert space $M_{4}\left(H_{W}\right) \otimes \mathbb{C}^{3}$ with the diagonal representation of the algebra. The only difference from the previous section is that the matrices $M_{l}$ and $M_{q}$ are no longer in $M_{2}(\mathbb{C})$ but in $M_{2}(\mathbb{C}) \otimes M_{3}(\mathbb{C})$. As the algebra acts diagonally on the Hilbert space (with respect to the generations), we can again repeat the arguments of Ref. [23] and argue that the $\operatorname{spin}_{c}$ condition will hold if algebras generated by $\pi_{L}(\mathcal{A})$ and $D_{l}, D_{q}$, respectively, will be full matrix algebras, that is, $\left(M_{4}(\mathbb{C}) \otimes\right.$ id $\otimes$ id $) \otimes M_{3}(\mathbb{C})$, independently for the lepton and for quarks.

Since the arguments we have used here are analogous to ones used in the discussion of full conditions (Section 4.2.2 in Ref. [23]), we infer the same condition for the Hodge property to be satisfied.

Both $M_{l}$ and $M_{q}$ can be diagonalized, yet because of the doublet structure of the left leptons and quarks, the components (up/down) cannot be diagonalized simultaneously. The standard presentation of the mass matrices for the physical standard model is then

$$
M_{l}=\left(\begin{array}{cc}
\Upsilon_{\nu} & 0 \\
0 & \Upsilon_{e}
\end{array}\right), \quad M_{q}=\left(\begin{array}{cc}
\Upsilon_{u} & 0 \\
0 & \Upsilon_{d}
\end{array}\right),
$$

where $\Upsilon_{e}$ and $\Upsilon_{u}$ are chosen diagonal with the masses of electron, muon, and tau and the up, charm, and top quarks, respectively, and

$$
\Upsilon_{\nu}=U \widetilde{\Upsilon_{\nu}} U^{\dagger}, \quad \Upsilon_{d}=V \widetilde{\Upsilon_{d}} V^{\dagger},
$$

with diagonal matrices $\widetilde{\Upsilon_{\nu}}, \widetilde{\Upsilon_{d}}$ providing (Dirac) masses of all neutrinos and down, strange, and bottom quarks, where $U$ is the Pontecorvo-Maki-Nakagawa-Sakata (PMNS) mixing matrix and $V$ is the Cabibbo-Kobayashi-Maskawa (CKM) mixing matrix.

As was indicated also in Ref. [23], the sufficient condition to fulfill the Hodge property is that for both pairs of matrices $\left(\Upsilon_{\nu}, \Upsilon_{e}\right)$ and $\left(\Upsilon_{e}, \Upsilon_{d}\right)$ their eigenvalues are pairwise different. This requirement is satisfied in the case of the physical standard model, provided that there is no massless neutrino (see Sec. 5.3 in Ref. [23]).

\section{From $\operatorname{spin}_{c}$ to Hodge condition}

Consider for a while the Hilbert space $H_{\mathrm{SM}}=M_{4}(\mathbb{C})$ with the same left and right representations of the algebra as in the standard model case (the standard model Hilbert space is the tensor product of the above with the space of Weyl fermions). Taken with the Krein-shifted Dirac $\widetilde{D_{F}}$ operator and $\Gamma=\pi_{L}(1,-1,1)$, it is a Euclidean even spectral triple.

Assume now that $\widetilde{D_{F}}$ is such that the $\operatorname{spin}_{c}$ condition holds. We shall describe now the procedure of the doubling of the triple so that the resulting real spectral triple satisfies the Hodge duality and is the finite spectral triple of the standard model studied so far as the finite component of the product geometry.

Consider $H_{\mathrm{SM}}^{2}=H_{\mathrm{SM}} \oplus H_{\mathrm{SM}}$ with the representation $\pi_{L} \oplus \pi_{R}$. We define the real structure $J$ as the composition of the Hermitian conjugation with the $\mathbb{Z}_{2}$ action exchanging the two copies of $H_{\mathrm{SM}}$, so that $J\left(M_{1} \oplus M_{2}\right)=M_{2}^{*} \oplus M_{1}^{*}$.

It is clear that the conjugation by $J$ maps the representation of the algebra $A$ to its commutant. We extend $\Gamma$ so that the relation $J \Gamma=\Gamma J$ holds and extend the Dirac operator $\widetilde{D_{F}}$ in the following way:

$$
D^{\prime}=\widetilde{D_{F}} \oplus 0+J\left(\widetilde{D_{F}} \oplus 0\right) J^{-1} .
$$

Clearly, $D^{\prime}$ anticommutes with $\Gamma$ and commutes with $J$. The Clifford algebra, that is, the algebra generated by $\pi_{L} \oplus$ $\pi_{R}$ and the commutators with $D^{\prime}$, is $C l_{\widetilde{D}_{F}}\left(\pi_{L}(A)\right) \oplus \pi_{R}(A)$. Because before the doubling we had the $\operatorname{spin}_{c}$ condition, it is clear that the commutant of the Clifford algebra contains $\pi_{R}(A) \oplus C l_{\widetilde{D}_{F}}\left(\pi_{L}(A)\right)$. It is therefore sufficient to verify that there are no other operators $T$ that map $H_{\mathrm{SM}}$ to $H_{\mathrm{SM}}$, which would satisfy that they commute with the representation of $C l_{D_{F}}\left(\pi_{L}(A)\right) \oplus \pi_{R}(A)$. Identifying the Hilbert space as $\mathbb{C}^{16} \oplus \mathbb{C}^{16}$, we see that the first component of Clifford algebra is $M_{4}(\mathbb{C}) \oplus M_{4}(\mathbb{C})^{(3)}$ (acting diagonally on $\mathbb{C}^{16}$; the notation $B^{(n)}$ means that we take $n$ copies of the algebra $B$ ), and the second is $\mathbb{C}^{(4)} \oplus M_{3}(\mathbb{C})^{(4)}$. Since all these algebras are independent of each other, there exists no operator intertwining their actions; hence, the commutant is exactly the one indicated above.

\section{Reality and the $C P$ violation}

Let us take the real structure $J$ acting on the finite part just by the complex conjugation, that is, the real structure implemented on $M_{4}\left(H_{W}\right)$ simply as id $\otimes \mathcal{J} \otimes \mathrm{id}$. Of course, it does not implement the usual zero-order condition; however, we still have a milder version of the zeroorder condition in the following form:

$$
\pi_{R}(\mathcal{A}) \subset J \pi_{L}(\mathcal{A}) J^{-1}
$$

We have already observed what are the commutation relations between $\tilde{D}$ and $\mathcal{J}$ (and hence $J$ ). Next, let us see whether similar commutation relations can be imposed on $\widetilde{D_{F}}$. As both $J^{2}$ as well as the anticommutation with $\Gamma$ are fixed, we see that by imposing the same KO-dimension (6) for the Euclidean finite spectral triple as for the Lorentzian spatial part we shall have $J \widetilde{D_{F}}=\widetilde{D_{F}} J$. 
This condition is very mild and means that the mass matrices $M_{l}$ and $M_{q}$ have to be real. In case of one generation of particles, it implies that masses of fermions have to be real, which is hardly very restrictive.

Yet the situation changes when we pass to three generations as already discussed above when considering the $\operatorname{spin}_{c}$ condition. Since $J$ acts by complex conjugation, then the requirement $\widetilde{D_{F}} J=J \widetilde{D_{F}}$ is still equivalent to the matrices $M_{l}, M_{q}$ having only real entries. Using the standard parametrization described above, this leads to the reality of the physical masses. However, since in the case of three generations the matrices $\Upsilon_{\nu}, \Upsilon_{d}$ are not diagonal, we must ensure that both $U$ and $V$ mixing matrices are real.

If this is the case, then all phases in the standard parametrization of these matrices should vanish, which physically will have the interpretation of the $C P$ symmetry preservation. However, in case of the CKM mixing matrix it implies that the Wolfenstein parameter $\bar{\eta}$ has to vanish, but experimentally, it is known that $\bar{\eta}=0.355_{-0.011}^{+0.012}$ [24].
The $C P$-violating phase $\delta_{\mathrm{CP}}^{\nu}$ in the neutrino sector, originated from the PMNS mixing matrix, was determined to be $\delta_{\mathrm{CP}}^{\nu} / \pi=1.38_{-0.38}^{+0.52}[24,25]$, which strongly confirms the $C P$ symmetry breaking. Therefore, the existence of $C P$ violation may be interpreted as a shadow of $J$-symmetry violation in the nondoubled spectral triple.

\section{E. Twisted (pseudo-Riemannian) spectral triple}

We have verified that the Krein-shifted Dirac operator satisfies the order-one condition (7). It appears that this is equivalent to the Lorentzian Dirac operator $D_{\mathrm{ST}}=\beta \widetilde{D_{\mathrm{ST}}}$ satisfying a twisted version of the order-one condition, that is,

$$
\left[\left[D_{\mathrm{ST}}, \pi_{L}(a)\right]_{\beta}, \pi_{R}(b)\right]_{\beta}=0,
$$

where $[x, y]_{\beta}=x y-\beta y \beta^{-1} x$ and $\beta=\mathrm{id} \otimes \gamma^{0} \otimes \mathrm{id}$. This follows directly from a simple computation, which uses $\beta^{2}=\mathrm{id}$ :

$$
\begin{aligned}
0 & =\left[\left[\widetilde{D_{\mathrm{ST}}}, \pi_{L}(a)\right], \pi_{R}(b)\right] \\
& =\beta D_{\mathrm{ST}} \pi_{L}(a) \pi_{R}(b)-\pi_{L}(a) \beta D_{\mathrm{ST}} \pi_{R}(b)-\pi_{R}(b) \beta D_{\mathrm{ST}} \pi_{L}(a)+\pi_{R}(b) \pi_{L}(a) \beta D_{\mathrm{ST}} \\
& =\beta\left(D_{\mathrm{ST}} \pi_{L}(a) \pi_{R}(b)-\beta \pi_{L}(a) \beta D_{\mathrm{ST}} \pi_{R}(b)-\beta \pi_{R}(b) \beta D_{\mathrm{ST}} \pi_{L}(a)+\beta \pi_{R}(b) \pi_{L}(a) \beta D_{\mathrm{ST}}\right) \\
& =\beta\left[\left[D, \pi_{L}(a)\right]_{\beta}, \pi_{R}(b)\right]_{\beta} .
\end{aligned}
$$

\section{CONCLUSIONS}

Let us stress that the geometry of the standard model, as discussed above, is not a product of spectral triples. Nevertheless, it has interesting features, which we summarize here with an outlook for the future research directions.

When restricted to the commutative algebra of realvalued functions (and its complexification), we obtain the even Lorentzian spectral triple with a real structure of KO-dimension 6 [compatible with the signature $(1,3)$ ] and with the Dirac operator satisfying the order-one condition.

On the other hand, the restriction of the spectral triple to the constant functions over the Minkowski space gives a Euclidean even spectral triple, which fails to be real. The failure of the real structure to satisfy the commutation relation with the (Krein-shifted) finite part of the Dirac operator is tantamount to the appearance of the violation of $C P$ symmetry in the standard model.

Neither of the restrictions satisfies the $\operatorname{spin}_{c}$ condition, as in both cases, we still consider the full Hilbert space. Yet the full spectral triple satisfies the $\operatorname{spin}_{c}$ condition in the following sense: the Clifford algebra generated by the commutators of the Krein-shifted Dirac operator with the representation $\pi_{L}$ of the algebra has, as the commutant, the right representation of the algebra $\pi_{R}$.

There are several possible ramifications of the above observations. First is the disappearance of the product structure; yet even if the triple is not a full product, then possibly it can have some structure of a quotient "spectral geometry." It will be interesting to classify all possible covers and all Dirac operators for them. In the presented spectral triple, the family of allowed Dirac operators that satisfy the $\operatorname{spin}_{c}$ condition is much closer to physical reality as it does not include any color symmetry-breaking operator unlike Ref. [7] and, moreover, the conditions are exactly the same as for the Hodge duality. The failure of the finite spectral triple to be real is then a geometric interpretation of the $C P$-symmetry breaking in the standard model. Finally, the disappearance of the product structure may have deep consequences for the spectral action. We postpone the discussion of possible effects on the physical parameters of the model for the forthcoming work. It will be interesting to compare the resulting Higgs mass (and other parameters) with both the experimental value and ones determined in other approaches. Further comparison with models going beyond the standard model, like the Pati-Salam model [26] (see also Ref. [27] for the link to pseudo-Riemannian structures), is also an interesting direction for future research.

\section{ACKNOWLEDGMENTS}

The authors thank L.Dąbrowski for helpful comments. 
[1] A. Connes, Noncommutative geometry and reality, J. Math. Phys. (N.Y.) 36, 6194 (1995).

[2] A. Connes, Gravity coupled with matter and foundation of non-commutative geometry, Commun. Math. Phys. 182, 155 (1996).

[3] A. H. Chamseddine, A. Connes, and M. Marcolli, Gravity and the standard model with neutrino mixing, Adv. Theor. Math. Phys. 11, 991 (2007).

[4] J. M. Gracia-Bondia, B. Iochum, and T. Schucker, The Standard Model in noncommutative geometry and fermion doubling, Phys. Lett. B 416, 123 (1998).

[5] F. D'Andrea, M. Kurkov, and F. Lizzi, Wick rotation and Fermion doubling in noncommutative geometry, Phys. Rev. D 94, 025030 (2016).

[6] M. Paschke, F. Scheck, and A. Sitarz, Can (noncommutative) geometry accommodate leptoquarks?, Phys. Rev. D 59, 035003 (1999).

[7] L. Dąbrowski, F. D'Andrea, and A. Sitarz, The Standard Model in noncommutative geometry: Fundamental fermions as internal forms, Lett. Math. Phys. 108, 1323 (2018).

[8] A. Bochniak and A. Sitarz, Finite pseudo-Riemannian spectral triples and the standard model, Phys. Rev. D 97, 115029 (2018).

[9] S. Farnsworth and L. Boyle, Non-commutative geometry, non-associative geometry and the standard model of particle physics, New J. Phys. 16, 123027 (2014).

[10] C. A. Stephan, Almost-commutative geometries beyond the standard model, J. Phys. A 39, 9657 (2006).

[11] C. A. Stephan, Almost-commutative geometries beyond the standard model. II. New colours, J. Phys. A 40, 9941 (2007).

[12] C. A. Stephan, New scalar fields in noncommutative geometry, Phys. Rev. D 79, 065013 (2009).

[13] A. H. Chamseddine and A. Connes, Resilience of the spectral standard model, J. High Energy Phys. 09 (2012) 104.
[14] A. Devastato, F. Lizzi, and P. Martinetti, Grand symmetry, spectral action, and the Higgs mass, J. High Energy Phys. 01 (2014) 042.

[15] A. Devastato, F. Lizzi, and P. Martinetti, Higgs mass in noncommutative geometry, Fortschr. Phys. 62, 863 (2014).

[16] A. Devastato and P. Martinetti, Twisted spectral triple for the Standard Model and spontaneous breaking of the grand symmetry, Math. Phys. Anal. Geom. 20, 2 (2017).

[17] G. Landi and P. Martinetti, On twisting real spectral triples by algebra automorphisms, Lett. Math. Phys. 106, 1499 (2016).

[18] A. Bochniak, A. Sitarz, and P. Zalecki (to be published).

[19] M. Paschke and A. Sitarz, Equivariant Lorentzian spectral triples, arXiv:mathph/0611029.

[20] S. Lord, A. Rennie, and J. C. Várilly, Riemannian manifolds in noncommutative geometry, J. Geom. Phys. 62, 1611 (2012).

[21] L. Dąbrowski and F. D'Andrea, The Standard Model in noncommutative geometry and Morita equivalence, J. Noncommut. Geom. 10, 551 (2016).

[22] R. Plymen, Strong Morita equivalence, spinors and symplectic spinors, J. Operator Theory 16, 305 (1986), https:// www.jstor.org/stable/24714803.

[23] L. Dąbrowski and A. Sitarz, Fermion masses, mass-mixing and the almost commutative geometry of the Standard Model, J. High Energy Phys. 02 (2019) 068.

[24] M. Tanabashi et al. (Particle Data Group), Review of particle physics, Phys. Rev. D 98, 030001 (2018).

[25] F. Capozzi, E. Di Valentino, E. Lisi, A. Marrone, A. Melchiorri, and A. Palazzo, Global contraints on absolute neutrino masses and their ordering, Phys. Rev. D 95, 096014 (2017).

[26] A. H. Chamseddine, A. Connes, and W. D. van Suijlekom, Grand unification in the spectral Pati-Salam model, J. High Energy Phys. 11 (2015) 011.

[27] A. Bochniak, T. E. Williams, and P. Zalecki, PseudoRiemannian structures in Pati-Salam models, arXiv:2002 .04506 . 